\title{
Adaptive Window Selection and Smoothing of Lomb Periodogram for Time-Frequency Analysis of Time Series
}

\author{
Shing-Chow Chan and Zhiguo Zhang \\ Department of Electrical and Electronic Engineering \\ The University of Hong Kong, Pokfulam Road, Hong Kong \\ scchan@eee.hku.hk, zgzhang@eee.hku.hk
}

\begin{abstract}
This article introduces a new adaptive Lomb periodogram for time-frequency analysis of time series, which are possibly non-uniformly sampled. It extends the conventional Lomb spectrum by windowing the observations and adaptively selects the window length by the intersection of confidence intervals (ICI) rule. To further reduce the variance of the Lomb periodogram due to time smoothing alone, time-frequency smoothing using local polynomial regression (LPR) is proposed. An orientation analysis is performed in order to derive a directional kernel in the time-frequency plane for adaptive smoothing of the periodogram. The support of this directional kernel is also adaptively selected using the ICI rule. Simulation results show that the proposed adaptive Lomb periodogram with timefrequency smoothing offers better time and frequency resolutions as well as lower variance than the conventional Lomb periodogram.
\end{abstract}

\section{INTRODUCTION}

Time-frequency distributions are useful tools for joint time-frequency analysis and visualization of signals. A conventional time-frequency distribution is the short-time Fourier transform (STFT) which is given by

$$
\operatorname{STFT}(t, \omega)=\int_{-\infty}^{\infty} x(\tau) w(\tau-t) e^{-j \omega \tau} d \tau,
$$

where $x(t)$ is the signal and $w(t)$ is the window function. The Fourier transform of $x(\tau) w(\tau-t)$ describes the local spectral content of $x(\tau)$ at a given time location $t$. The support of the window $w(t)$ is crucial to the analysis because it determines the bias and variance trade-off of the STFT.

For sinusoidal components in the signal, a long time window is desirable because more samples can be used to determine their amplitudes around the given time location. Hence, the variances of the spectral coefficients are reduced and better frequency resolution is obtained. On the contrary, for fast time-varying components in the signals, a smaller window support is preferred in order to reduce the bias in estimating these components. As a result, a better time resolution is achieved for tracking these fast changing components. However, a small window support also reduces the frequency resolution of other slowly varying frequency components. Therefore, a fundamental problem of timefrequency analysis is the design of the windows in order to achieve the best bias-variance trade-off in estimating the various components. In [1], Jones and Parks address the biasvariance tradeoff problem in STFT by using an adaptive window in the time plane and the concept of "concentration measure". The basic idea is to select the parameters of a series of local windows with different time and frequency supports in order to maximize the "concentration measure". Another interesting approach is the adaptive window selection approach of Katkovnik and Stankovic [3, 4], where the window size is selected by the intersection of confidence intervals (ICI) rule. It has been successfully applied to estimate the instantaneous frequency (IF) of a time-varying component for the Wigner distribution [3] and the $M$ periodogram [4]. Recently, Hussain and Boashash [6] further developed this algorithm to multi-components signal. However, their algorithm is based on the IF estimation to choose optimal window size only at time instant $t$, not at frequency instant $\omega$.

In this paper, we consider the problem of time-frequency analysis of time series, which are possibly non-uniformly sampled. Our approach is based on a novel extension of the Lomb periodogram $[7,8]$, which is a conventional method for carrying out spectral analysis of non-uniformly spaced samples. Lomb periodogram has been adapted in many areas such as biomedical sciences, astronomy and finance. Its basic idea is to estimate the amplitude of a given sinusoid with a certain angular frequency $\omega$ by least squares fitting the sinusoidal to the observed non-uniform data points. The power of this sinusoid is then denoted by Lomb periodogram $P(\omega)$. One significant drawback of the Lomb periodogram is that it is obtained by fitting the whole data block to the signal model. Therefore, its tracking ability is severely limited. In order to track time-varying signals and produce a timefrequency Lomb spectrum or distribution, we first propose a windowed version of the Lomb periodogram. To achieve the best bias-variance trade off, the support of this window is optimally selected at each time instant and frequency by the ICI rule. This significantly improves the time-frequency resolution of the windowed Lomb spectrogram. To further reduce the variance of the new distribution, which is also a problem in most time-window based distributions, smoothing in the time-frequency plane using local polynomial regression (LPR) is proposed. In order to exploit the time-frequency localization of the adaptive Lomb periodogram, an orientation analysis is performed in order to derive a directional kernel in the time-frequency plane for adaptive smoothing of the periodogram. The support of this directional kernel is also adaptively selected using the ICI rule. Simulation results show that the proposed adaptive Lomb periodogram with time-frequency smoothing offers better time and frequency 
resolution as well as lower variance than the conventional Lomb periodogram.

The paper is organized as follows: in Section 2, the windowed Lomb periodogram is introduced. The adaptive Lomb periodogram and window selection using the ICI rule are introduced in Section 3. Section 4 is devoted to the orientation analysis and directional filtering of the time-frequency distribution (TFD). Simulation results and comparisons are described in Section 5. Finally, conclusions are drawn in Section 6.

\section{Windowed Lomb Periodogram}

The Lomb method $[7,8]$ is a very useful method for spectral analysis of non-uniform sampled data. The basic idea of the Lomb periodogram is to estimate the amplitude of a given sinusoid with a certain angular frequency $\omega$ by least squares fitting the sinusoidal to the observed non-uniform data points. The power of this sinusoid is then denoted by $P(\omega)$. In other words, $P(\omega)$ is computed from the observed data points using a least-squares fit to the model:

$$
x(t)=A \cos (\omega t+\theta)+n(t),
$$

where $n(t)$ denotes the additive noise and other components in the signal. Since the Lomb method weights the data on a "per point" basis instead of on a "per time interval" basis, it can be applied to non-uniform data.

The detailed computation can be summarized as follows: given a set of non-uniform discrete-time observation $x_{i}=x\left(t_{i}\right) ; i=1,2, \ldots N$, the mean $\bar{x}$ and variance $\sigma^{2}$ of the data are first computed.

To evaluate the Lomb spectrum at an angular frequency $\omega=2 \pi f>0$, a least squares fit to the sinusoid in (5) is performed on the zero mean and normalized observations. It yield the following Lomb normalized periodogram:

$$
\begin{aligned}
& P(\omega)=\frac{\left\{\sum_{i}\left(x_{i}-\bar{x}\right) \cos \omega\left(x_{i}-\tau\right)\right\}^{2}}{2 \sigma^{2} \sum_{i} \cos ^{2} \omega\left(x_{i}-\tau\right)} \\
&+\frac{\left\{\sum_{i}\left(x_{i}-\bar{x}\right) \sin \omega\left(x_{i}-\tau\right)\right\}^{2}}{2 \sigma^{2} \sum_{i} \sin ^{2} \omega\left(x_{i}-\tau\right)},
\end{aligned}
$$

where $\tau$ is given by

$$
\tan (2 \omega \tau)=\frac{\sum_{i} \sin 2 \omega t_{i}}{\sum_{i} \cos 2 \omega t_{i}} .
$$

The constant $\tau$ is an offset that makes the periodogram invariant to time translation. It can be seen that the conventional Lomb spectrum is obtained by fitting the whole data block of $N$ samples to the signal model. Therefore, its tracking ability is severely limited. In order to track time-varying signals and produce a time-frequency Lomb spectrum or distribution, we need to include a window, which should be ideally adapted to the observation to achieve the best biasvariance tradeoff. To this end, we introduce a window $w\left(t_{i}\right)$, which is centred at time $t$, so that we can calculate the Lomb periodogram on the windowed data $x^{\prime}\left(t_{i}\right)=x\left(t_{i}\right) w\left(t_{i}-t\right)$ at a given time instant $t$. Hence, we can obtain a series of pe- riodograms (or more precisely a TFD) $P(t, \omega)$ of the signal $x(t)$ at different time locations $t$. This yields

$$
\begin{aligned}
& P(t, \omega)= \frac{\left\{\sum_{i}\left(x^{\prime}\left(t_{i}\right)-\overline{x^{\prime}}\right) \cos \left(\omega\left(x^{\prime}\left(t_{i}\right)-\tau\right)\right)\right\}^{2}}{2 \sigma^{\prime 2} \cdot \sum_{i} \cos ^{2}\left(\omega\left(x^{\prime}\left(t_{i}\right)-\tau\right)\right)} \\
&+\frac{\left\{\sum_{i}\left(x^{\prime}\left(t_{i}\right)-\overline{x^{\prime}}\right) \sin \left(\omega\left(x^{\prime}\left(t_{i}\right)-\tau\right)\right)\right\}^{2}}{2 \sigma^{\prime 2} \cdot \sum_{i} \sin ^{2}\left(\omega\left(x^{\prime}\left(t_{i}\right)-\tau\right)\right)},
\end{aligned}
$$

where $\bar{x}$ is the mean of the data in the window $w\left(t_{i}\right), \sigma^{\prime}$ is the standard deviation of the data in the window $w\left(t_{i}\right)$. Similar to STFT, the selection of window length is crucial to the time-frequency resolution of the Lomb TFD $P(t, \omega)$. Again, for a long window, the frequency resolution for sinusoids will improve, while the time resolution of the varying components will be reduced, and vice versa. Motivated by the novel bandwidth selection rule in [5], we propose to use the ICI rule for determining the window size of $w\left(t_{i}\right)$ at each time $t$. Also, to reduce the variance of the frequency distribution, smoothing in the frequency domain using local polynomial regression is also performed as suggested in [9] for convention periodogram (which is not time-varying). Finally, directional smoothing in the time-frequency plane will be performed to cater for time varying components. The details will be given in the following sections.

\section{Adaptive Lomb Periodogram and Window Size Selection}

Now, we will employ the ICI rule to determine the window length of the Lomb periodogram at every point $(t, \omega)$ in the time-frequency plane. Suppose that we are given a set of finite window sizes in ascending order of magnitude:

$$
H=\left\{h_{j} \mid h_{1}<h_{2}<\ldots<h_{j}\right\} .
$$

For each window size $h_{j}$, we obtain a Lomb periodogram $P_{h,}(t, \omega)(j=1, \ldots, J)$. The variance, $\operatorname{Var}(\cdot)$, and the bias, bias $(\cdot)$, of these Lomb periodograms are functions of the window $h_{j}$, so is the mean square error (MSE):

$$
\operatorname{MSE}\left(t, \omega ; h_{j}\right)=\operatorname{Var}\left[P_{h_{i}}(t, \omega)\right]+\operatorname{bias}^{2}\left[P_{h_{i}}(t, \omega)\right] .
$$

We know that the variance and bias are respectively decreasing and increasing functions of the window size. Therefore, there exists an optimal window $h_{v p t}$ such that the two terms are equal and $\operatorname{MSE}\left(t, \omega ; h_{j}\right)$ is minimized. To determine this optimal bandwidth, the $\mathrm{ICl}$ rule examines a sequence of confidence intervals of the estimates $P_{h_{t}}(t, \omega)$ :

$$
\begin{aligned}
& D_{j}=\left[L_{j}, U_{j}\right], \\
& U_{j}=P_{h_{i}}(t, \omega)+\Gamma \cdot \sigma\left(h_{j}\right), \\
& L_{j}=P_{h_{i}}(t, \omega)-\Gamma \cdot \sigma\left(h_{j}\right),
\end{aligned}
$$

where $\sigma\left(h_{i}\right)$ is the variance of the estimate using the window with length $h_{i}$, and $\Gamma>0$ is a threshold parameter of the confidence interval. Define the following quantities from the confident intervals 


$$
\begin{aligned}
& \bar{L}_{j}=\max \left[\bar{L}_{j-1}, L_{j}\right], \underline{U}_{j}=\min \left[\underline{U}_{j-1}, U_{j}\right], \\
& j=1,2, \cdots, J, \bar{L}_{0}=\underline{U}_{0}=0 .
\end{aligned}
$$

The largest value of these $j$ for which $\underline{U}_{j} \geq \bar{L}_{j}$ gives $j^{+}$ and it yields a bandwidth $h_{j}{ }^{+}$, which is the desired optimal bandwidth. In other words, the optimal bandwidth $h_{j}{ }^{+}$is the largest $j$ when $\underline{U}_{j} \geq \bar{L}_{j}$ is still satisfied. By using an adaptive window for each sinusoid and at each time $t$, better bias and variance trade off can be achieved for multi-component with different time-frequency variations. Hence, a better performance in time-frequency resolution is obtained.

Laguna, Moody and Mark [10] make clear that in fact the Lomb periodogram is the $\left|X_{i}(f)\right|^{2}$ spectrum, where $X_{i}(f)$ is the DFT of the unevenly sampled signal $x_{i}^{\prime} \cdot x_{i}^{\prime}$ is defined as:

$$
x_{i}^{\prime}=\frac{1}{\sqrt{N}} \sum_{i=1}^{N} x(t) \delta\left(t-t_{i}\right)
$$

So, the values of Lomb periodogram are correlative with the window sizes. Before we select the optimal Lomb periodogram values from different periodograms with different window sizes, a normalization process must be applied. The normalization method is to divide the Lomb periodogram by its corresponding window size.

\section{ORIENTATION ANALYSIS AND DIRECTIONAL SMOOTHING OF PERIOdOGRAMS}

The adaptive Lomb periodogram (TFD) we obtained in Section 3 has a significantly better time frequency resolution than the conventional Lomb spectrum with fixed time windows (as we shall demonstrate later at section 5). However, it is well known that the variance of the periodogram at each frequency is still very high. This can be seen as spurious components and blurring in the adaptive Lomb periodogram, for example in Figure 1(c). One method to reduce this variance is to smooth the periodogram in the frequency domain [9]. Although there are many methods for smoothing a 2D signal, for example using a low-pass 2D filter, it is still desirable to achieve the best bias-variance tradeoff so that both over- and under- smoothing can be avoided. This can be achieved by performing a $2 \mathrm{D}$ local polynomial regression (LPR) to the TFD using again the ICI rule for adaptive bandwidth selection. However, the shape or orientation of the window plays a important role during the smoothing. Most work considered for multidimensional LPR considered only isotopic window, which is symmetry in all the dimensions. Clearly, this is undesirable in TFD because the spectrum components are usually localized in the time-frequency plane.

To overcome this problem, we propose to employ a directional window and adaptively vary its window size using the ICI rule to achieve better time-frequency resolution. To determine the orientation of the window at a certain point on the time-frequency plane, an orientation analysis is first performed using a set of filters with different orientations. Since the output energy of a filer at a given orientation is measure of local energy of the TFD at the given orientation, by using a sufficient fine angular resolution, we can identify different orientations of the TFD at a given location from the maximal values of the energy plot. These directions are also estimates of the instantaneous frequencies and chirp rates of the TFD at a point $(t, \omega)$. For simplicity, we have used 32 orientations between 0 and $\pi / 2$, and then interpolated the signal to a finer grid for locating the maxima.

After we get the directions of every point in the timefrequency domain, we can use a directional Gaussian filter to smooth the periodogram using LPR. During directional smoothing of the TFD, the window lengths of the directional filters are determined again by the ICI rule to achieve better bias-variance tradeoff. In general, more complicated nonlinear directional smoothing can be applied. For the present work, the frequency of a component is assumed to be a linear function of time $t$. In other words, for frequency component that varies nonlinearly with time, we are essentially approximating them locally by a straight line over a short time interval. More research will be left for future work Finally, we remarked that in time-frequency analysis, it is usually desirable to depict the TFD on an evenly sampled time-frequency plane. For non-uniformly spaced observations, only spectrums at non-evenly sampled points in time coordinate are available. So, it is necessary to interpolate the periodogram to a uniform grid for visualization purpose. This can be achieved after the directional smoothing of the TFD using 2D LPR.

\section{EXPERIMENTS RESULTS}

Example 1: Our first example is an evenly sampled monocomponent nonlinear frequency modulated signal. The IF of the signal is:

$$
\omega(n T)=0.08 \pi \cdot \operatorname{asinh}(n T-50)+0.5 \pi .
$$

The time interval is $T=1$, and the number of points are from $n=0,1,2 \cdots 100$. The signal is assumed to be corrupted by additive Gaussian noise and the SNR is $10 \mathrm{~dB}$. The windows lengths considered are 16, 32, 64, and 128. Fig. 1 shows the Lomb periodogram obtained by using fixed as well as the adaptive window lengths. We can see that when the window length is small, the time resolution is high but the frequency resolution is low, while when the window is long, the frequency resolution is high but the time resolution is low. The proposed adaptive Lomb periodogram with adaptive window is able to achieve good time as well as frequency resolutions. From the adaptive window length, it can be seen that when there are fast changes in the frequency component, a small window is selected to strive for a better time-resolution. On the hand, when the signal changes slowly, a long time window will be selected for better frequency resolution. However, we can see that the variance of the adaptive Lomb spectrum in Fig.2(a) is still considerable because of the limited performance of using just a time window for smoothing as in many other approaches [6]. After using the proposed directional smoothing in the time-frequency domain, a much better performance is obtained as shown in Fig.2 (b). Detail examination of Fig.2 also reveals that the amplitude fluctua- 
tion (blurring) of the signal component is much less for the proposed directional TF smoothing than adaptive time smoothing alone. After the directional smoothing, the variance of the periodogram at the instantaneous frequency point changes from 8.72 to 7.58 . Since the frequency model we used is a linear function of time while the signal is nonlinear frequency modulated, there is still some blurring of the periodogram. In all cases, the ICI rule is used to select the filter length of the TFD.
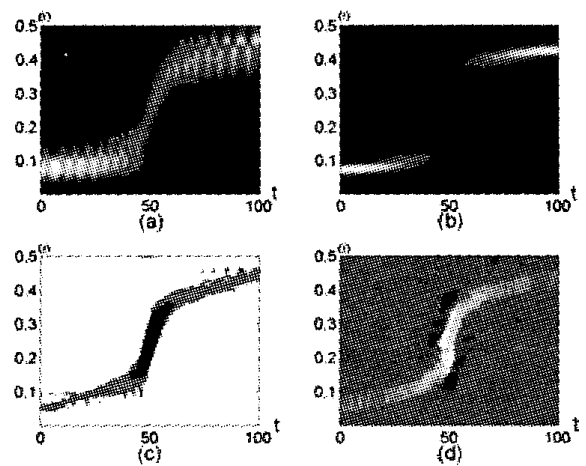

Fig. 1: Windowed Lomb periodogram with (a) $h=16,(b) h=128$, (c) adaptive window lengths, (d) local periodogram orientation, the pixel values become bigger when angles (from 0 to $\pi / 2$ )

become larger.
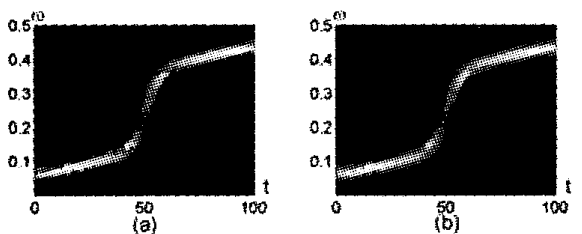

Fig. 2 (a) Lomb periodogram with adaptive time window, (b) adaptive periodogram with directional TF smoothing.

Example 2: Our next example is a non-evenly sampled multi-component linear FM signal given by

$$
\begin{aligned}
& \varphi_{1}(n T)=0.008 n T+0.1, \\
& \varphi_{2}(n T)=-0.008 n T+0.9 .
\end{aligned}
$$

The time interval is $T=0.5$, and the observation is in the time interval $n=0,1,2 \cdots, 200$. The non-uniform observation is obtained by randomly sampling 100 points from these observations, so the average interval is $2 T=1$. Again, the signal is assumed to be corrupted by additive Gaussian noise and the SNR is $10 \mathrm{~dB}$. The possible window lengths are again chosen from the set $\{16,32,64,128\}$. We can see the periodogram can detect frequency which is larger than $\mathrm{Ny}$ quist frequency $1 /(2 T)=0.5$. After the directional smoothing, the variance of the periodogram at the instantaneous frequency point is lowered from 1.10 to 0.48 . This is because the frequency components of the signal is changing linearly with time, so the improvement of smoothing are more significant than the last example.
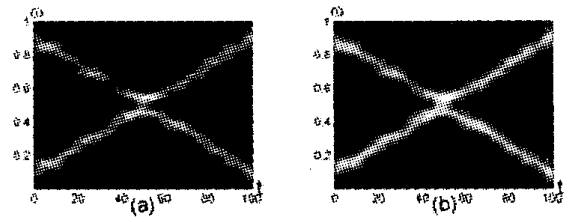

Fig. 3: (a) non-uniform Lomb periodogram with adaptive time window, (b) adaptive periodogram with directional TF smoothing and time interpolation.

\section{Conclusion}

This paper has presented a new adaptive Lomb periodogram for time-frequency analysis of time series, which are possibly non-uniformly sampled. It extends the conventional Lomb spectrum by using an adaptive directional timefrequency window. It addresses the bias-variance tradeoff problem by means of a directional window with varying time-frequency support, which is selected by the ICI rule. Simulation results show that the proposed adaptive Lomb periodogram with time-frequency smoothing offers better time and frequency resolutions as well as lower variance than the conventional Lomb periodogram. The time-frequency smoothing is also applicable to reduce the variance of related periodograms such as the STFT.

\section{REFERENCES}

[1] D.L. Jones and T.W. Parks, "A high resolution data- adaptive time-frequency representation", IEEE Trans. ASSP, Vol. 38, No 12, pp. 21272135 , Dec. 1990.

[2] V. Katkovnik, "On adaptive local polynomial approximation with varying bandwidth", IEEE ICASSP 1998, Seattle, Washington, United States of America, 1998.

[3] L.J. Stankovic and V. Katkovnik, "The Wigner distribution of noisy signals with adaptive time-frequency varying window", IEEE Trans. on Signal Processing, pp.1099-1108, Vol. 47, No. 2, Apr. 1999.

[4] I. Djurovic, V. Katkovnik and L.J. Stankovic, "Instantaneous frequency estimation based on the robust spectrogram", IEEE ICASSP 2001, Salt Lake City, Vol.6, pp.3517-3520, May 2001.

[5] L.J. Stankovic, V. Katkovnik, "Algorithm for the instantaneous frequency estimation using time-frequency distributions with adaptive window width", Signal Processing Letters, IEEE, Vol. 5, Issue 9, pp. 224-227, Sept. 1998.

[6] Z.M. Hussain and B. Boashash, "Adaptive instantaneous frequency estimation of multicomponent FM signals using quadratic time-frequency distributions", IEEE Trans. on Signal Processing. pp.1866-1876, Vol. 50, No. 8, Aug.2002.

[7] N.R. Lomb, "Least-squares frequency analysis of unequally spaced data", Astrophysics and Space Science. Vol. 39, pp. 447$462,1976$.

[8] W.H. Press, B.P. Flannery, S.A. Teukolsky, and W.T. Vetterling, Numerical recipes in C, Cambridge Univ. Press, 1988.

[9] T.C.M. Lee, "A stabilized bandwidth selection method for kernel smoothing of the periodogram", Signal Processing, pp.419430 , Vol.81, 2001.

[10] P. Laguna, G. Moody, and R.G. Mark, "Power spectral density of unevenly sampled data by least-square analysis: performance and application to heart rate signals", IEEE Trans. on Biomedical Engineering, pp. 698 - 715, Vol. 45, Issue 6, June 1998 